\title{
Study of distributed algorithm for water sources monitoring based on wireless sen- sor networks
}

\author{
Haifeng Zhang ${ }^{1}$, Xiaowei Liu ${ }^{1}$, Long Chen ${ }^{1}$, Xianhui Shao ${ }^{1}$, Hui Luan ${ }^{1}$ \\ ${ }^{1}$ MEMS Center, Harbin Institute of Technology, Harbin, Heilongjiang, China
}

\begin{abstract}
In order to improve the coverage of wireless sensor networks in the environment of water quality monitoring, the paper proposes a distributed algorithm which is based on diamond-shaped virtual force. In this algorithm, the differences between measurements and current calculated distances are modeled into forces, dragging the nodes close to their actual positions. Based on the diamond method, the algorithm can determine the predefined threshold of virtual force and optimize the global distribution. Compared with the simulation of existing algorithms, it is proved to have superior performance.
\end{abstract}

Keywords: Wireless sensor network, Diamond virtual force, Network coverage, Threshold.

\section{Introduction}

With the rapid economic development, pollution and destruction of water is increasingly serious. As one of the primary means of water resources management, water environmental monitoring is playing an irreplaceable role ${ }^{[1]}$. Reasonable distribution of4wireless sensor network can improve the coverage and connectivity of wireless sensor networks, and can reduce energy consumption and cost of network $^{[2,3]}$.

In this paper, a distributed algorithm which is based on diamond virtual force is putted forward. It solves the problem of traditional algorithm which is not adaptive to meet different measurements. Diamond virtual force algorithm can adapt to the distributed environment which contains obstacles and hotspots ${ }^{[4]}$. And the search performance is still good with the sensor size increasing.

\section{Diamond virtual force algorithm}

The mode of wireless sensor node measurement includes binary and probabilistic measurement models. Due to the imprecision of binary model, we select probabilistic measurement model. Assume that the location of the wireless sensor node si is (xi, yi) and the target is located in (x, y), then the distance between the target and si is given as Eq. 1 .

$$
d\left(s_{i}, P\right)=\sqrt{\left(x_{i}-x\right)^{2}+\left(y_{i}-y\right)^{2}}
$$

Probability measurement model ${ }^{[5]}$ of $s_{i}$ is given by Eq. 2 .

$$
c_{x y}\left(s_{i}\right)=\left\{\begin{array}{cc}
0 & r+r_{e} \leq d\left(s_{i}, P\right) \\
e^{\left(-\alpha_{1} \lambda_{1}^{\beta}\left(1 \lambda_{2}^{\beta \beta_{2}}+\alpha_{2}\right)\right.} & r-r_{e}<d\left(s_{i}, P\right)<r+r_{e} \\
1 & d\left(s_{i}, P\right) \leq r-r_{e}
\end{array}\right.
$$

Where $r_{e}\left(0<r_{e}<r\right)$ is reliability metrical parameter of wireless sensor nodes, $\alpha_{1}, \alpha_{2}, \beta_{1}, \beta_{2}$ is used for features measure of wireless sensor nodes, input parameters $\lambda_{1}, \lambda_{2}$ are defined as follows Eq. 3, Eq. 4. 


$$
\begin{aligned}
& \lambda_{1}=r_{e}-r+d\left(s_{i}, P\right) \\
& \lambda_{2}=r_{e}+r-d\left(s_{i}, P\right)
\end{aligned}
$$

When re is larger than zero, the metrical process requires multiple wireless sensor nodes. The probability of joint measurement is given by Eq5.

$$
c_{x, y}\left(S_{o v}\right)=1-\prod_{s_{i} \in S_{o v}}\left(1-c_{x, y}\left(s_{i}\right)\right)
$$

Where $S_{o v}$ is the aggregate of wireless sensor nodes to be measured, assume that $c_{t h}$ is the probability threshold of wireless nodes, the effective measurement condition is that the least probability of joint measurement is less than $c_{t h}$.

$$
\min _{x, y}\left\{c_{x, y}\left(s_{i}, s_{j}\right)\right\} \leq c_{t h}
$$

Algorithm of virtual force assumes that all kinds of objects in wireless sensor networks can produce unequal force to wireless node. According to the forces, wireless sensor nodes move a corresponding distance until them reach the force balance. The forces it suffered are given by Eq. 7 .

$$
\vec{F}_{i}=\sum_{j=1, j \neq i}^{k} \vec{F}_{i j}+\vec{F}_{i R}+\vec{F}_{i A}
$$

Where $\vec{F}_{i}$ is total virtual force which is received by wireless node $\mathrm{s}_{\mathrm{i}}, \vec{F}_{i j}$ is the force from sensor $j$ to sensor $i, \vec{F}_{i R}$ is the force from obstacle $R$ to sensor $i, \vec{F}_{i A}$ is the force from hot zone $A$ to sensor $i$. $\vec{F}_{i j}$ is given by Eq. 8 .

$$
\overrightarrow{F_{i j}}=\left\{\begin{array}{cr}
0 & d_{i j} \geq C \\
\left(w_{A}\left(d_{i j}-d_{t h}\right), \alpha_{i j}\right) & C>d_{i j}>d_{t h} \\
0 & d_{i j}=d_{t h} \\
\left(w_{R}\left(\frac{1}{d_{i j}}-\frac{1}{d_{t h}}\right), \alpha_{i j}+\pi\right) & d_{i j}<d_{t h}
\end{array}\right.
$$

Where $\mathrm{C}$ is the communication radius of sensor node, $d t h$ is the distance threshold, $d_{i j}$ is the distance of two sensors, $\alpha_{i j}$ is the azimuth of wireless sensor nodes, $\omega_{A}$ is the gravitational coefficient, $\omega_{R}$ is the repulsive coefficient.

After the analysis of virtual force, the wireless nodes begin to move according the size and direction of virtual force. They are given by Eq. 9, Eq.10.

$$
\begin{aligned}
& x_{\text {new }}=\left\{\begin{array}{c}
x_{\text {old }} \quad\left|F_{x y}\right| \leq F_{t h} \\
x_{\text {old }}+\frac{F_{x}}{F_{x y}} \times \operatorname{MaxStep} \times e^{\frac{-1}{F_{y y}}}\left|F_{x y}\right|>F_{t h}
\end{array}\right. \\
& y_{\text {new }}=\left\{\begin{array}{cc}
y_{\text {old }} & \left|F_{x y}\right| \leq F_{\text {th }} \\
y_{\text {old }}+\frac{F_{y}}{F_{x y}} \times \operatorname{MaxStep} \times e^{\frac{-1}{F_{y y}}} & \left|F_{x y}\right|>F_{\text {th }}
\end{array}\right.
\end{aligned}
$$

Where $F_{t h}$ is the force threshold, $F_{x y}$ is the total forces, $F_{x}$ is the x-axis component of total virtual force, $F_{y}$ is the $y$-axis component of total virtual force, MaxStep is the max removable distance.

When the distance threshold is too small, the distribution is dense and coverage can not be guaranteed; when the distance threshold is too large, the sparse distribution is easy to form blind spots. Diamond algorithm can determine the best distance threshold. It can be seen from Fig. 1.

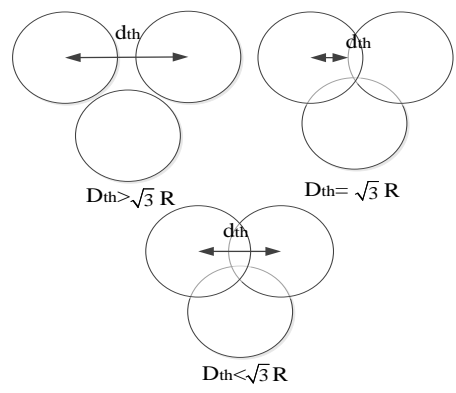

Fig 1. Compare of distance threshold

Assume that the distance of each center of a circle is dth and the radius of cir- 
cle is $\mathrm{R}$, it can be seen that the best distance threshold is $\sqrt{3} \mathrm{R}$.

\section{Results and discussion}

32 wireless sensor nodes are distributed in the square measurement area $(80 \times 80 \mathrm{~m})$. The parameters of simulation are given by Table 1 .

Table 1. Parameters of simulation

\begin{tabular}{|l|l|l|l|l|l|}
\hline $\mathrm{R}[\mathrm{m}]$ & $\mathrm{C}[\mathrm{m}]$ & $\omega_{A}$ & $\omega_{R}$ & $\begin{array}{l}\mathrm{Max} \\
\text { step[ } \\
\mathrm{m}]\end{array}$ & \multirow{2}{*}{} \\
\hline 7 & 21 & 1 & 5 & 3.5 & \\
\hline$r_{e}[m]$ & $\alpha_{1}$ & $\alpha_{2}$ & $\beta_{1}$ & $\beta_{2}$ & $C_{t h}$ \\
\hline 3.5 & 1 & 0 & 1 & 0.5 & 0.9 \\
\hline
\end{tabular}

According to the table, the best predefined threshold of the diamond algorithm is $12 \mathrm{~m}$. Wireless sensor network nodes distribution of random initialization is given by Fig. 2a. The distribution of wireless sensor network nodes which is optimized by the virtual force is given by Fig. 2b, Fig.2c Fig. 2d when the predefined threshold is $10 \mathrm{~m}, 14 \mathrm{~m}, 12 \mathrm{~m}$.
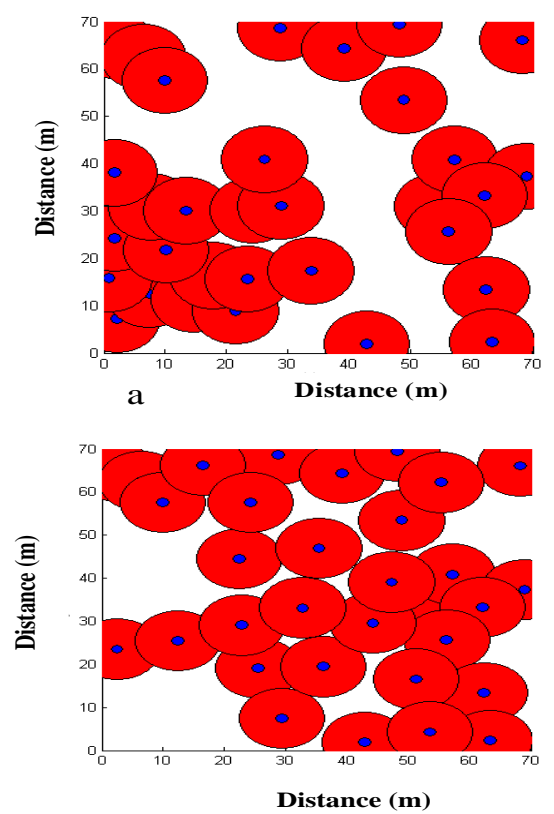

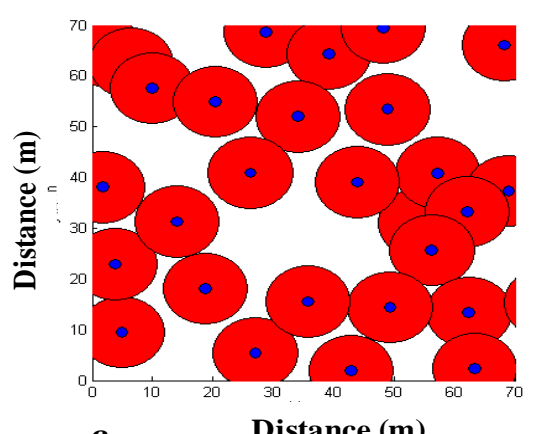

c Distance (m)

Fig. 4 Predefined threshold $=14 \mathrm{~m}$

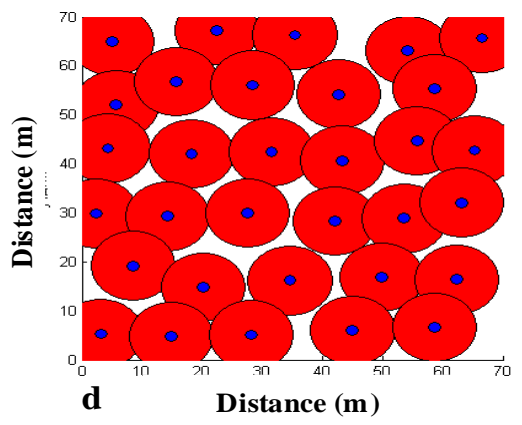

a: Random initialization, b: Predefined threshold $=10 \mathrm{~m}$. c: Predefined threshold $=14 \mathrm{~m}, \mathrm{~d}$ : Predefined threshold $=12 \mathrm{~m}$

Fig. 2 network coverage of different Predefined threshold

Wireless sensor network coverage of Fig. $2 \mathrm{a}$ is $59.22 \%$, the coverage of Fig. 2b is $75.07 \%$, the coverage of Fig. $2 \mathrm{c}$ is $69.30 \%$, the coverage of Fig. $2 \mathrm{~d}$ is $90.99 \%$. When the predefined threshold equals to $12 \mathrm{~m}$, it can get the largest network coverage.

Diamond virtual force algorithm can also be applied to the network coverage of the environment containing obstacles and hotspots. Predefined threshold equal to $12 \mathrm{~m}$ the network coverage is shown in Fig.3. Here, green area stands for the obstacles, yellow area stands for the hotspots. From the Fig.3 we can see that the hotspots totally covered by the network signal, the network signal can be 
transmitted over obstacles. Signals can be evenly cover the entire detection region.

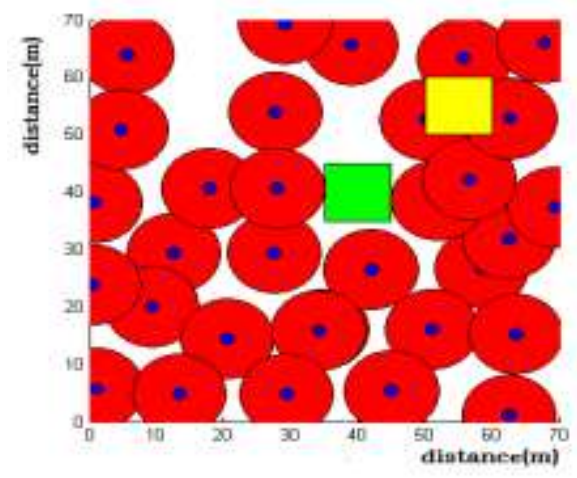

Fig .3 Network distribution for the hotspots and obstacle based on Diamond virtual force algorithm

\section{Conclusion}

This paper presents an optimization algorithm which is based on diamond virtual force. The algorithm is applied for the distribution of wireless sensor nodes in the environment of water quality monitoring. It solves the problem of choosing predefined threshold, and provides a valuable reference for distributing wireless sensor nodes in the area of water sources.

\section{Acknowledgement}

The authors would like to acknowledge the financial support from the national high technology research and develop ment program of china (Grant No. SS 2012AA062902), Major Science and Technology Program for Water Pollution Control and Treatment (Grant No. 2012ZX07201003-01), Key Science and Technology Project of Harbin (Grant No. 2011AA1CG003).

\section{References}

[1] W. Xue, W. Sheng, "An improved particle filter for target tracking in sensor system," J. Sensors, Vol.7, pp. 144-156, 2007.

[2] W. Xue, J. Aiguo, W. Sheng, "Mobile agent based wireless sensor network for intelligent maintenance," J. Lecture Notes in Computer Science, pp. 316 - 325, 2005.

[3] K. Chakrabarty, Y. Zou, "Sensor deployment and target localization in distributed sensor networks," J. ACM Transactions on Embedded Computing Systems (TECS), pp. 61-91,2004.

[4] Mayur C. Akewar, Nileshsingh V. Thakur, "A Study of Wireless Mobile Sensor Network Deployment", International Journal of Computer Networks and Wireless Communications, Vol(2), pp.533-541,2012.

[5] Y. Zou, K. Chakrabarty, "Sensor deployment and target localization based on virtual forces," $C$. IEEE INFOCOM, Vol(21), pp. 1293 - 1303, 2003. 